\title{
Klimawandel als strafrechtlicher Notstand
}

\section{Zugleich Besprechung des Urteils des Bezirksgerichts Lausanne PE19.000742/PCL/llb vom 13. Januar 2020}

\section{Andrés Payer *}

Das Urteil befasst sich mit der Frage, ob im Rahmen von Klimaprotesten verübte Straftaten unter den rechtfertigenden Notstand (Art.17 StGB) fallen, und es bejaht nun dies im konkreten Fall. Es hält nach Ansicht des Autors einer näheren Überprüfung nicht stand und kann im Ergebnis auch nicht durch notstandsähnliche Institute des Strafrechts gestützt werden. Es ist hingegen der Strafbefreiungsgrund des fehlenden Strafbedürfnisses (Art. 52 StGB) anwendbar.

I. Einleitung

II. Rechtfertigender Notstand (Art. 17 StGB)

1. Notstandsfähige Rechtsgüter ...........................................................229

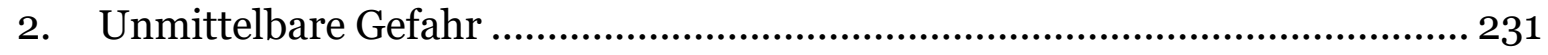

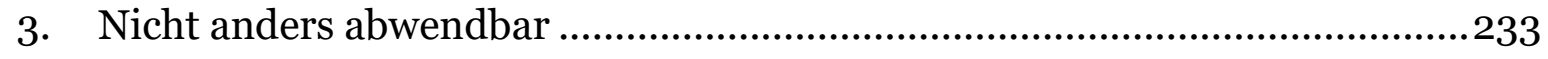

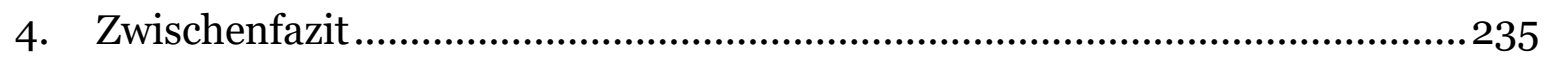

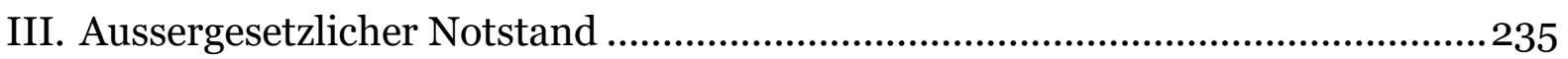

IV. Wahrnehmung berechtigter Interessen ..................................................236

V. Zur Möglichkeit des Absehens von der Strafe.............................................239

Zitiervorschlag: Andrés Payer, Klimawandel als strafrechtlicher Notstand, in: suigeneris 2020, S. 226

URL: $\quad$ sui-generis.ch/132

DOI: $\quad$ https://doi.org/10.21257/sg.132

* Andrés Payer (andres.payer@rwi.uzh.ch), Wissenschaftlicher Hilfsassistent am Lehrstuhl für Strafrecht, Strafprozessrecht und Internationales Strafrecht der Universität Zürich von Prof. Dr. Felix Bommer, Juristischer Mitarbeiter bei Isenring Melunovic Kessler Kuhn Partner, Meilen. 


\section{Einleitung}

1 Dass sich Aktivisten, die unerlaubte Protestaktionen durchführen, auf einen entlastenden Notstand oder Zwang zum Handeln berufen, hat im internationalen Kontext eine lange Tradition. ${ }^{1}$ Das war etwa der Fall bei Aktionen gegen die Verwendung von Atomwaffen ${ }^{2}$ oder verbotene Aktivitäten durch Geheimdienste3, und auch im Bereich des Umweltschutzes, wie namentlich beim Protest gegen die Rodung des AmazonasRegenwaldes 4 oder die Luftverschmut-

1 Vgl. nachfolgend Fn. 2-5. Zu beachten ist, dass die verschiedenen Rechtsordnungen den Notstand an unterschiedliche Voraussetzungen knüpfen und diesem eine andere dogmatische Stellung einräumen können als dies im hiesigen Strafbarkeitsaufbau der Fall ist (vgl. etwa zum Notstand im US-amerikanischen Recht Jochen Etzel, Notstand und Pflichtenkollision im amerikanischen Strafrecht, Diss. Freiburg i.Br. 1993, S. 41 ff., 95 ff., 120 ff.; Sybille Marti, Rechtsvergleichendes über den rechtfertigenden Notstand - Die Schweiz, Deutschland und die U.S.A. im Vergleich, in: Martino Mona/Kurt Seelmann (Hrsg.), Grenzen des rechtfertigenden Notstands, Zürich 2006, S. 41 ff., 52 ff.; Niklaus Schmid, Strafverfahren und Strafrecht in den Vereinigten Staaten: eine Einführung, 2. Aufl., Heidelberg 1993, S. $216 \mathrm{ff}$.).

2 Urteil des Columbia County District Court (Oregon, USA) 77-246 ff. vom 16. Dezember 1977 (State v. Mouer); Robert Aldridge/Virginia Stark, Nuclear War, Citizen Intervention, and the Necessity Defense, Santa Clara Law Review 1986, S. 299 ff., 310 f.; Lance N. Long/Ted Hamilton, Case Comment - Washington v. Brockway: One Small Step Closer to Climate Necessity, McGill Journal of Sustainable Development Law, 2017, S. 151 ff., 159; dies., The Climate Necessity Defense: Proof and Judicial Error in Climate Protest Cases, Stanford Environmental Law Journal 2019, S. 57 ff., 74 f.

3 Urteil des Northampton County District Court (Massachusetts, USA) vom 15. April 1987, zit. in: Matthew L. Wald, «Amy Carter Is Acquitted Over Protest», The New York Times Nr. 47,111 vom 16. April 1987, S. 17.

4 Urteil der Sala Penal de Apelaciones Transitoria y Liquidadora de Bagua (Bagua, Peru) 00194-2009 (0163-2013) vom 22. September 2016; Urteil des Juzgado Mixto de la provincia de Datém del Ma- zung5, ist der Notstand geltend gemacht worden. Vor diesem Hintergrund mag es wenig überraschen, wenn nun vermehrt auch Klimaaktivisten, die im Einsatz für einen stärkeren Klimaschutz Strafbestimmungen verletzen, den Notstand anrufen. ${ }^{6}$ Wenn sie damit aber hierzulande Erfolg haben und für sich auf dieser Grundlage einen Freispruch erringen, so überrascht dies dann doch, wie die Reaktionen auf das Urteil des Bezirksgerichts Lausanne PE19.000742/PCL/llb vom 13. Januar 2020 (im Folgenden: «Urteil BGL») gezeigt haben.7

rañón (Loreto, Peru) 2008-00109-0-1903-SPPE-2 vom 10. Dezember 2009.

5 Urteil des Criminal Court of the City of New York (New York, USA) 571 N.Y.S.2d $851 \mathrm{ff}$. vom 14. März 1991 (People v. Gray); John Alan Cohan, Civil Disobedience and the Necessity Defense, The University of New Hampshire Law Review, S. 111 ff., 121; Long/Hamilton, Standford Environmental Law Journal 2019 (Fn. 2), S. 75 f.

6 Vgl. Urteil des Washington Supreme Court (Washington, USA) No. 97182-o vom 4. September 2019 (State v. Ward); Urteil des Washington Court of Appeals (Washington, USA) No. 77044-6-I vom 4. August 2019 (State v. Ward); ferner das derzeit in Washington, USA, laufende Verfahren gegen den Klimaaktivisten George E. Taylor, den 122 Rechtsprofessoren mit Eingabe ans Appellationsgericht vom 14. Januar 2020 unterstützt haben.

7 Allein in der NZZ sind in den ersten Wochen nach der Publikation des Urteils eine ganze Reihe von Beiträgen erschienen, in denen die durch das Urteil BGL ausgesprochene Anwendbarkeit des rechtfertigenden Notstands auf den Klimaprotest kontrovers erörtert wurde, vgl. Antonio Fumagalli, «Ein historisches Urteil», NZZ Nr. 10 vom 14. Januar 2020, S. 11; dens., «Überraschender Sieg für Klimaaktivisten», NZZ Nr. 10 vom 14. Januar 2020, S. 13; Lucien Scherrer, «Richter im Aktivistenmodus», NZZ Nr. 11 vom 15. Januar 2020, S. 11; Hansueli Schöchli, «Das würde zu Chaos führen», NZZ Nr. 11 vom 15. Januar 2020, S. 13; Daniel Gerny/Claudia Baer, «Rechtfertigt politischer Protest Straftaten?», NZZ Nr. 11 vom 15. Januar 2020, S. 13; Antonio Fumagalli, «Der Richter, der die Klimaaktivisten freisprach», NZZ Nr. 13 vom 17. Januar 2020, S. 13; Andrea Kučera, «Der Richter, der alle überrascht», NZZaS Nr. 3 vom 19. Januar 2020, S. 9; Martin Rhonheimer, «Der Aufrechte rechnet mit Strafe», NZZ 
2 Besagtem Urteil lag - in aller Kürze folgender Sachverhalt zugrunde: Eine Gruppe von 20 bis 30 Klimaaktivisten hatte eine Filiale der Bank Crédit Suisse in Lausanne besetzt, um die Teilhabe des Tennisspielers Roger Federer am öffentlichen Image der «Schweizer Bank mit der schlechtesten $\mathrm{CO}_{2}$-Bilanz» 8 zu denunzieren. Als Tennisspieler verkleidet simulierten die Aktivisten, Tennis zu spielen und rollten ein Banner mit der Aufschrift «Crédit Suisse détruit le climat. Roger, tu cautionnes ça? \#SiRogersavait» aus. Dabei blockierten sie die Kunden der Bank zwar nicht, behinderten aber deren Zugang zu den Bankomaten. Ein Teil der Aktivisten widersetzte sich weiter der Aufforderung der Polizei, die Filialräume zu verlassen.

Das Bezirksgericht Lausanne hatte infolgedessen zu beurteilen, ob sich zwölf der Aktivisten des Hausfriedensbruchs und des Widerstands gegen polizeiliche Anordnungen strafbar gemacht hatten. Dies waren die einzigen Vorwürfe, die den Aktivisten zur Last gelegt wurden. Ob das Verfahren noch auf andere Straftatbestände hätte ausgedehnt werden müssen (z.B. die Nötigung), entzieht sich der Kenntnis des aussenstehenden Betrachters. Das Bezirksgericht Lausanne sprach die Angeklagten von allen Vorwürfen wegen rechtfertigenden Notstands (Art. 17 StGB) frei.

Nr. 19 vom 24. Januar 2020, S. 37. Das Urteil hat darüber hinaus international hohe Wellen geworfen, vgl. stellvertretend Christopher Borucki, Rechter ziet klimaatverstoring als rechtvaardiging voor burgerlijke ongehoorzaamheid (Tribunal de police Lausanne 13 januari 2020, PE19.000742/PCL/LLB), De Juristenkrant 2020, S. $6 \mathrm{f}$.

8 Vgl. die Presseerklärung der hinter der Protestaktion stehenden «Lausanne Action Climat» sowie Urteil BGL, S. 25, 27, 31, 34 .
4 Einen ähnlichen Fall hatte kurze Zeit später das Polizeigericht Genf zu beurteilen. Diesmal ging es um Aktivisten, die mit rot angemalten Händen - als Symbol für die Opfer des Klimawandels - die Fassade einer Bankfiliale der Crédit Suisse befleckt hatten. Einer der Aktivisten wurde in der Folge wegen Sachbeschädigung angeklagt. Er wandte vor Gericht ein, in einem rechtfertigenden Notstand im Sinne des Art. 17 StGB gehandelt zu haben. Das Polizeigericht Genf kam allerdings zum Schluss, dass die Voraussetzungen des rechtfertigenden Notstands nicht erfüllt seien; die Anwendung des Art. 17 StGB scheitere schon am Erfordernis einer unmittelbaren, nicht anders abwendbaren Gefahr. Es verurteilte deshalb den Angeklagten zu einer bedingten Geldstrafe und einer Busse. ${ }^{9}$

5 Gegen beide Urteile, sowohl dasjenige des Bezirksgerichts Lausanne als auch jenes des Polizeigerichts Genf, ist Berufung eingelegt worden. Streitpunkt ist vor allem die Anwendung bzw. Nichtanwendung des rechtfertigenden Notstands auf die jeweiligen Klima-Aktionen. Somit sind in den beiden Fällen diesbezüglich weitere Diskussionen absehbar. Ausserdem sind aufgrund der hohen Aktualität des Klimathemas weitere KlimaProtestaktionen und weitere juristische Auseinandersetzungen zu solchen Aktionen zu erwarten.

6 Mit den nachfolgenden Ausführungen soll nun einmal prinzipiell der Frage nachgegangen werden, ob und gegebenenfalls unter welchen Voraussetzungen im Rahmen von Klimaprotesten verübte Straftaten nach geltendem Recht vom

$9 \longdiv { \text { Urteil des Polizeigerichts Genf } \mathrm { P } / 2 4 1 2 3 / 2 0 1 8 }$ vom 20. Januar 2020. 
rechtfertigenden Notstand (Art. 17 StGB) erfasst sind und, falls nicht, ob andere, notstandsähnliche Institute des Strafrechts in solchen Fällen einer Bestrafung im Wege stehen können. ${ }^{10}$

\section{Rechtfertigender Notstand (Art. 17 StGB)}

7 Schon begriffsnotwendig setzt der rechtfertigende Notstand (Art. 17 StGB) eine Notstandslage voraus. Diese besteht bekanntlich in einer Interessenkollision, bei der eine unmittelbare Gefahr für ein bestimmtes Rechtsgut nur durch die Begehung einer strafbewehrten Tat abgewandt werden kann.

\section{Notstandsfähige Rechtsgüter}

8 Daraus ergibt sich zunächst die Frage, welche Rechtsgüter notstandsfähig sind: Sind dies nur die Güter des Individuums wie Leib, Leben und Freiheit oder fallen darunter auch kollektive Rechtsgüter wie der Bestand des Staates oder die rechtsstaatlich-demokratische Ordnung ${ }^{11}$ ? Der Wortlaut des Art. 17 StGB indiziert ersteres, indem er von der Begehung einer mit Strafe bedrohten Tat «um ein eigenes oder das Rechtsgut einer andern Person [...] zu retten» («pour préserver [...] un bien juridique lui appartenant ou appartenant à un tiers»/ «per preservare un bene giuridico proprio o un bene giuri-

10 Nicht behandelt werden Irrtumskonstellationen wie z.B. der Putativnotstand oder der indirekte Verbotsirrtum. Diesbezüglich sei auf die einschlägige Literatur verwiesen, vgl. stellvertretend Günter Stratenwerth, Schweizerisches Strafrecht, Allgemeiner Teil I: Die Straftat, 4. Aufl., Bern 2011, § $10 \mathrm{~N} 112 \mathrm{ff}$.

11 Vgl. Ioanna Anastasopoulou, Deliktstypen zum Schutz kollektiver Rechtsgüter, Diss. München 2005, S. 28; Roland Hefendehl, Kollektive Rechtsgüter im Strafrecht, Habil. München, Köln et al. 2002, S. 19. dico altrui») spricht, und die Gesetzesmaterialien bestätigen, dass dies dem Willen des historischen Gesetzgebers entspricht. ${ }^{12}$ Die Beschränkung des rechtfertigenden Notstands auf den «individuellen Notstand» kann zudem aus der Funktion der Notrechte abgeleitet werden: Sie sollen den Schutz der Rechtsgüter des Individuums ermöglichen, wenn keine staatliche Hilfe präsent ist, dem Einzelnen aber nicht das Recht geben, als eine Art Hilfspolizist tätig zu werden. ${ }^{13}$ Es ist somit im Einklang mit der herrschenden Meinung ${ }^{14}$ festzustel-

$12 \overline{\text { Vgl. Botschaft vom 21. September } 1998 \text { zur Ände- }}$ rung des Schweizerischen Strafgesetzbuches (Allgemeine Bestimmungen, Einführung und Anwendung des Gesetzes) und des Militärstrafgesetzes sowie zu einem Bundesgesetz über das Jugendstrafrecht, BBl 19991979 ff., S. 2006; Carl Stooss, Bericht über den Vorentwurf zu einem Schweizerischen Strafgesetzbuch nach den Beschlüssen der Expertenkommission, Bern 1899, S. 45 f.; ders., Motive zu dem Vorentwurf eines Schweizerischen Strafgesetzbuches, Basel/Genf 1893, S. 32 ff.; vgl. ferner zum StGB vor der ATRevision 2002 Philipp Thormann/Alfred v. Overbeck, Das Schweizerische Strafgesetzbuch, Bd. I, Zürich 1940, Art. 34 N 5 ff.; zur Entwicklung des ersten StGB von 1937 Ernst Brägger, Der Notstand im Schweizerischen Strafrecht, Diss. Bern, Aarburg 1937, S. 93 ff.; Ernst Hafter, Lehrbuch des Schweizerischen Strafrechts, Allgemeiner Teil, Berlin 1926, S. 147 f.

13 Gunther Arzt, Kleiner Notstand bei kleiner Kriminalität?, in: Andreas Donatsch/Niklaus Schmid (Hrsg), Strafrecht und Öffentlichkeit, Festschrift für Jörg Rehberg zum 65. Geburtstag, Zürich 1996, S. 25 ff., 29 ff.; Helmut Frister, Strafrecht Allgemeiner Teil, 8. Aufl., München 2018, 16. Kap. N 6, 17. Kap. N 2; anders Walter Perron, in: Albin Eser et al. (Hrsg.), Strafgesetzbuch, Schönke/Schröder Kommentar, 30. Aufl., München 2019, § 34 N 10 (zit. S/S-K dStGBAutor).

14 BGE 94 IV 68 E. 2; Arzt (Fn. 13), S. 29 ff.; Anna Coninx, Das Solidaritätsprinzip im Lebensnotstand, Diss. Bern 2012, S. 249; Andreas Donatsch, in: Andreas Donatsch (Hrsg.), StGB JStG - Kommentar, 20. Aufl., Zürich 2018, Art. $17 \mathrm{~N} 2$ (zit. OFK StGB-Autor); ders./Brigitte Tag, Strafrecht I, Verbrechenslehre, 9. Aufl., Zürich/Basel/Genf 2013, S. 239 f.; Michel Dupuis et al., CP, Code pénal, Petit commentaire, 2. Aufl., 
len, dass im Lichte des Art. 17 StGB nur Individualrechtsgüter notstandsfähig sind.

9 Genauer zu erörtern ist vor diesem Hintergrund die Argumentation des Bezirksgerichts Lausanne im Urteil BGL, wonach «Par exception, la protection d'un bien juridique collectif sera toutefois pertinente sous l'angle de cette disposition [Art. 17 StGB] si un bien juridique

Basel 2017, Art. 17 N 13; Philippe Graven/Bernhard Sträuli, L'infraction pénale punissable, 2. Aufl., Bern 1995, N 93; José Hurtado Pozo/Thierry Godel, Droit pénal général, 3. Aufl., Zürich/Basel/Genf 2019, N 714; Nikolai Iwangoff, Die Duldungspflichten im rechtfertigenden Notstand, Diss. Basel, Basel/Genf/München 2009, S. 13 f., 131 ff.; Gilles Monnier, in: Robert Roth/Laurent Moreillon (Hrsg.), Code pénal I, Commentaire romand, Basel 2009, Art. $17 \mathrm{~N} 13$ (zit. CR CP I-Autor); Marcel Alexander Niggli/Carola Göhlich, in: Marcel Alexander Niggli/Hans Wiprächtiger (Hrsg.), Strafrecht, Basler Kommentar, Bd. I, 4. Aufl., Basel 2019, Art. 17 N 5 (zit. BSK StGB IAutor); Umberto Pajarola, Gewalt im Verhör zur Rettung von Menschen, Diss. Zürich, Bern et al. 2007, S. 297; Franz Riklin, Schweizerisches Strafrecht, Allgemeiner Teil I, Verbrechenslehre, 3. Aufl., Zürich/Basel/Genf 2007, § 14 N 45; Grace Schild Trappe, Verkehrsregelverletzungen und Notstandslage, in: Assista TCS SA (Hrsg.), Collezione Assista, Genf 1998, S. 640 ff., 644 ff.; dies./Felix Schöbi, Not kennt kein Gebot beim Hausfriedensbruch?, Jusletter 11. Mai 2020, N 16; Hans Schultz, Einführung in den Allgemeinen Teil des Strafrechts, Bd. I, 4. Aufl., Bern 1982, S. 164; Kurt Seelmann/Christopher Geth, Strafrecht Allgemeiner Teil, 6. Aufl., Basel 2016, N 162; Stratenwerth (Fn. 10), § 10 N 41; Stefan Trechsel/Christopher Geth, in: Stefan Trechsel/Mark Pieth (Hrsg.), Schweizerisches Strafgesetzbuch, Praxiskommentar, 3. Aufl., Zürich/St. Gallen 2018, Art. 17 N 4 (zit. PK StGBAutor); Stefan Trechsel/Peter Noll/Mark Pieth, Schweizerisches Strafrecht Allgemeiner Teil I, 7. Aufl., Zürich/Basel/Genf 2017, S. 116; Wolfgang Wohlers, in: Wolfgang Wohlers/Gunhild Godenzi/Stephan Schlegel (Hrsg.), Schweizerisches Strafgesetzbuch, Handkommentar, 4. Aufl., Bern 2020, Art. 17 N 3 (zit. HK StGB-Autor); anders O.A. Germann, Das Verbrechen im neuen Strafrecht, Zürich 1942, Art. 34 N 1/5; sowie offenbar (laut Donatsch/Tag [Fn. 14], S. 239, wohl unbeabsichtigt) BGE 106 IV 65 E. 4. personel est également en jeu».15 Diese Aussage ist insofern zutreffend, als eine Gefährdung von Rechtsgütern der Allgemeinheit oft zugleich eine unmittelbare Gefahr für ein Individualrechtsgut beinhaltet, sodass dann aus diesem Grund eine Notstandslage besteht. ${ }^{16}$ Das ändert aber nichts daran, dass kollektive Rechtsgüter als solche nicht notstandsfähig sind. Soweit also das Bezirksgericht Lausanne die Erhaltung des Klimas und der Umwelt als Allgemeingüter qualifiziert und unter dem Aspekt des rechtfertigenden Notstands (Art. 17 StGB) berücksichtigt, 17 ist dem entgegenzuhalten, dass es bei Art. 17 StGB nur um den Schutz von Individualrechtsgütern geht. Abgesehen davon ist unklar, ob es sich beim Schutz des Klimas und der Umwelt überhaupt um Rechtsgüter handelt; dies kann jedenfalls hierzulande nicht als anerkannt gelten. ${ }^{18}$

15 Urteil BGL, S. 49, E22. 4.1, S. 55, E. 4.2, im Anschluss an CR CP I-Monnier (Fn. 14), Art. 17 $\mathrm{N}$ 13. Die Aussage findet sich zudem bei Dupuis et al. (Fn. 14), Art. $17 \mathrm{~N} 13$.

16 Vgl. Frister (Fn. 13), 17. Kap. N 2; Arnaud Nussbaumer, L'aquittement des activistes du climat à Lausanne, in: lawinside; Max Waiblinger, SJK 1958, Nr. 1205, Ziff. 7. Das hängt damit zusammen, dass die Güter des Individuums stets auch mittelbar der Allgemeinheit, und die Güter der Allgemeinheit stets mittelbar dem Einzelnen dienen (Knut Amelung, Rechtsgüterschutz und Schutz der Gesellschaft, Diss. Göttingen, Frankfurt a.M. 1972, S. 207 ff., 388 ff.; Anastasopoulou [Fn. 11], S. 40; Winfried Hassemer, Theorie und Soziologie des Verbrechens, Habil. Frankfurt a.M. 1973, S. 231 ff.; Hefendehl [Fn. 11], S. 60 ff.).

17 Urteil BGL, S. 54 f., E. 4.2.

18 Vgl. zur im Schrifttum um das Rechtsgut «Umwelt» geführte Kontroverse die Nachweise bei Jürg-Beat Ackermann/Kerstin Schröder Bläuer, in: Marcel Alexander Niggli/Hans Wiprächtiger (Hrsg.), Strafrecht, Basler Kommentar, Bd. II, 4. Aufl., Basel 2019, Art. 230 $0^{\text {bis }} \mathrm{N} 3$ (zit. BSK StGB II-Autor). Eine selbstständige Erörterung dieser Frage muss hier ausgespart blieben, um den angemessenen Rahmen nicht zu sprengen. 
Nach dem Gesagten ist festzuhalten, dass es sich beim Schutz des Klimas und der Umwelt nicht um notstandsfähige Rechtsgüter handelt. Es bleibt die Frage, ob das Interesse an der Erhaltung des Klimas und der Umwelt womöglich unter sog. aussergesetzliche Rechtfertigungsgründe fallen, zu denen gemäss Bundesgericht «u.a. der übergesetzliche Notstand und die Wahrnehmung berechtigter Interessen» gehören. ${ }^{19}$ Sie wird später zu erörtern sein (unten III. und IV.).

Mit Bezug auf den rechtfertigenden Notstand ist jedenfalls erforderlich, dass Individualrechtsgüter gefährdet sind, damit dieser Rechtfertigungsgrund zur Anwendung gelangt. Dabei kommen beliebige Güter des Einzelnen infrage, und es ist unbeachtlich, wer Träger des betroffenen Rechtsguts ist. ${ }^{20}$ Im vorliegenden Kontext könnten - angesichts der prognostizierten Konsequenzen des Klimawandels (namentlich das Auftauen des Permafrosts, der Anstieg der Meeresspiegel, die Versauerung der Meere, die Zunahme von Hitzewellen und extremen Wetterund Klimavorkommnissen, die fortschreitende Wüstenbildung und die weitere Verbreitung von Schädlingen und Krankheitserregern) ${ }^{21}$ - insbesondere die Individualgüter Leib, Leben, Gesundheit, Freiheit, Eigentum und Vermögen gefährdet sein (vgl. unten II.B.). Der recht-

BGE 94 IV 68 E. 2.

20 Botschaft (Fn. 12), BBl 19991979 ff., S. 2005 f.

21 Vgl. Ove Hoegh-Guldberg et al., Chapter 3, Impacts of $1.5^{\circ} \mathrm{C}$ Global Warming on Natural and Human Systems, in: Valérie Masson-Delmotte et al. (Hrsg.), Global Warming of $1.5^{\circ} \mathrm{C}$, An IPCC Special Report on the impacts of global warming of $1.5^{\circ} \mathrm{C}$ above pre-industrial levels and related global greenhouse gas emission pathways, in the context of strengthening the global response to the threat of climate change, sustainable development, and efforts to eradicate poverty, Genf 2018, S. 175 ff., 240 ff., 263 m.w.N. fertigende Notstand fällt mithin nicht von vornherein ausser Betracht.

\section{Unmittelbare Gefahr}

12 Vorausgesetzt ist, dass sich ein (Individual-)Rechtsgut in unmittelbarer Gefahr befindet. Eine Gefahr im Sinne des Art. 17 StGB liegt vor, wenn eine Wahrscheinlichkeit der Rechtsgutsverletzung besteht, ${ }^{22}$ wobei völlig abstrakte Risiken hier ausscheiden müssen; dagegen liegt eine Gefahr auch dort vor, wo es um Jedermanns-Gefahren geht, sofern sie sich im Einzelfall konkretisieren. ${ }^{23}$ Irrelevant ist die Quelle der Gefahr, d.h. ob sie von einem Menschen ausgeht oder nicht, z.B. von einem Naturereignis. ${ }^{24} \mathrm{Ob}$ eine Gefahr besteht, muss ex ante und aus Sicht eines verständigen Dritten in der Lage des Täters eingeschätzt werden. ${ }^{25}$ Unmit-

22 Vgl. Urteil des Obergerichts Obwalden vom 12. März 2002, in: AbR 2002/03 Nr. 25 E. 2.a/c/aa/aaa; Dupuis et al. (Fn. 14), Art. 17 N 6; Graven/Sträuli (Fn. 14), N 90; Hurtado Pozo/Godel (Fn. 14), N 708; CR CP I-Monnier (Fn. 14), Art. 17 N 6; PK StGB-Trechsel/Geth (Fn. 14), Art. 17 N 3.

23 Vgl. Günther Jakobs, Strafrecht Allgemeiner Teil, 2. Aufl., Berlin/New York 1991, 13. Abschn. N 12; Kristian Kühl, Strafrecht, Allgemeiner Teil, 8. Aufl., München 2017, § 8 N 40; Florian Richter, Rechtfertigender Notstand zur Erhaltung von Arbeitsplätzen?, Diss. München, Berlin et al. 2019, S. 288.

24 Donatsch/Tag (Fn. 14), S. 241; Dupuis et al. (Fn. 14), Art. 17 N 6; Graven/Sträuli (Fn. 14), N 91; Iwangoff (Fn. 14), S. 12; CR CP I-Monnier (Fn. 14), Art. 17 N 6; Seelmann/Geth (Fn. 14), N 162; Stratenwerth (Fn. 10), § 10 N 42; PK StGBTrechsel/Geth (Fn. 14), Art. 17 N 3; Trechsel/ Noll/Pieth (Fn. 14), S. 115.

25 Brägger (Fn. 12), S. 142; Coninx (Fn. 14), S. $264 \mathrm{ff}$; Anton Eberle, Die Bedeutung der Selbsthilfe im Strafrecht, Diss. Bern 1951, S. 51; Iwangoff (Fn. 14), S. 13 ff.; Martino Mona/ Nicolas Leu, Tücken der Notwehr - Abirrungen, Irrtümer und allerlei Gefahren für unbeteiligte Dritte bei Notwehrhandlungen, recht 2011, S. 175 ff., 186; BSK StGB I-Niggli/Göhlich (Fn. 14), Art. 17 N 11; Seelmann/Geth (Fn. 14), N 163; PK StGB-Trechsel/Geth (Fn. 14), Art. 17 N 3. 
telbar ist die Gefahr nach der herrschenden Meinung nicht nur, wenn der Schadenseintritt unmittelbar bevorsteht, sondern auch dann, wenn eine Abwehr später nicht mehr oder nur noch unter sehr viel grösseren Risiken möglich wäre. ${ }^{26} \mathrm{Es}$ geht also bei der Unmittelbarkeit der Gefahr weniger um die zeitliche Entfernung des Schadenseintritts als vielmehr um den Zwang zur Entscheidung und die Notwendigkeit sofortigen Handelns. ${ }^{27}$

Im besprochenen Fall erblickte das Bezirksgericht Lausanne die Gefahr in den Konsequenzen des Klimawandels und verwies hierzu vor allem auf die Berichte des Weltklimarats (IPCC), ${ }^{28}$ in denen seitens zahlreicher Klimatologen namentlich vor dem Auftauen des Permafrosts, dem Anstieg der Meeresspiegel, der Versauerung der Meere, der Vermehrung von Hitzewellen und extremen Wetterund Klimavorkommnissen, der fortschreitenden Wüstenbildung (Desertifikation) und der weiteren Verbreitung von Schädlingen und Krankheitserregern eindringlich gewarnt wird.29 Mit diesen

$26 \overline{\text { Vgl. }}$ BGE 125 IV 49 E. 2; $\quad$ BGE 122 IV 1 E. 3; BGE 98 IV 5; BGE 75 IV 49; Iwangoff (Fn. 14), S. 16; BSK StGB I-Niggli/Göhlich (Fn. 14), Art. 17 N 14; Seelmann/Geth (Fn. 14), N 164; Stratenwerth (Fn. 10), § 10 N 42; PK StGB-Trechsel/ Geth (Fn. 14), Art. 17 N 5.

27 Michael Pawlik, Der rechtfertigende Notstand, Berlin/New York 2002, S. 177; S/S-K dStGBPerron (Fn. 13), § 34 N 17; Richter (Fn. 23), S. $289 \mathrm{f}$.

28 Urteil BGL, S. 50, E. 4.2.

29 Vgl. die Nachweise in Fn. 21; ferner Myles R. Allen et al., Chapter 1, Framing and Context, in: Masson-Delmotte et al. (Fn. 21), S. 49 ff., 51, 66; Heleen de Coninck et al., Chapter 4, Strengthening and Implementing the Global response, in: Masson-Delmotte et al. (Fn. 21), S. 313 ff., 319; Ove Hoegh-Guldberg et al. (Fn. 21), S. 177, 276 f.; Joeri Rogerlj et al., Chapter 2, Mitigation Pathways Compatible with $1.5^{\circ} \mathrm{C}$ in the Context of Sustainable Development, in: Masson-Delmotte et al. (Fn. 21), S. 93 ff., 142, 146; je m.w.N.
Klimafolgen sind zwangsläufig auch Beeinträchtigungen der Individualgüter Leib, Leben, Gesundheit, Freiheit, Eigentum, Vermögen etc. verbunden.30 Dabei handelt es sich nicht bloss um eine ganz entfernte oder nur gedankliche Möglichkeit des Schadenseintrittes, sondern um bereits jetzt (auch hierzulande) spürbare Folgen des Klimawandels, wie sich etwa am Beispiel der Gemeinde Randa (VS) zeigt, deren Einwohner durch die Schmelze eines Hängegletschers am Weisshorn bedroht sind,31 oder im Engadin, das wegen der Schmelze des Permafrosts einem höheren Risiko für Felsstürze und Schlammlawinen ausgesetzt ist. ${ }^{2}$ Es kann daher festgehalten werden, dass eine durch den Klimawandel bedingte Wahrscheinlichkeit der Verletzung von Individualgütern besteht, welche - soweit konkretisiert im Einzelfall - eine Gefahr im Sinne von Art. 17 StGB darstellt.

14 Was das Kriterium der Unmittelbarkeit der Gefahr anbetrifft, so kommen die vorstehend zitierten Klimatologen zum Schluss, dass sofortige Klimaschutzmassnahmen nötig seien bzw. der letztmögliche Zeitpunkt gekommen sei, um die schädlichen Konsequenzen des Klimawandels für die betroffenen Individualrechtsgüter sicher abzuwehren.33 Diese Einschätzung bekräftigte auch die vom Bezirksgericht Lausanne befragte Profes-

30 Vgl. die Nachweise in Fn. 21 und 29.

${ }^{31}$ Siehe hierzu die lokale Berichterstattung sowie die Reportagen des SRF «Un glacier toujours plus instable menace les habitants de Randa (VS)», «Randa VS: Ein Hängegletscher bedroht das Dorf», «Das Dorf, das dem Hängegletscher trotzt».

32 Linda Koponen/Larissa Rhyn, Die Gefahr lauert im Berg, NZZ Nr. 264 vom 13. November 2019, S. $14 \mathrm{f}$.

33 Vgl. die Nachweise in Fn. 21 und 29. 
sorin für Klimatologie an der ETH Zürich, Sonia Senivaratne.34 In Anbetracht dessen und vor dem Hintergrund der vorherrschenden extensiven Auslegung des Kriteriums der Unmittelbarkeit der Gefahr erscheint die Ansicht des Bezirksgerichts Lausanne, es liege aufgrund der drohenden Konsequenzen des Klimawandels eine unmittelbare Gefahr im Sinne von Art. 17 StGB für die Rechtsgüter Leben und Gesundheit vor, 35 vertretbar. ${ }^{36}$ Es ist indes zu präzisieren, dass erstens weitere Individualrechtsgüter als die im Urteil BGL genannten und zweitens (soweit sich die Gefahr im Einzelfall konkretisiert) nicht bloss die Güter der Aktivisten selbst, sondern aller Rechtsgutsträger betroffen sind, zumal Art. 17 StGB auch die Notstandshilfe erfasst.

\section{Nicht anders abwendbar}

15 Geht man davon aus, dass die Konsequenzen des Klimawandels eine unmittelbare Gefahr für Individualrechtsgüter darstellen (vgl. oben II.B.), so muss diese Gefahr für eine Rechtfertigung nach Art. 17 StGB zudem nicht anders $a b$ wendbar sein als durch die Begehung der jeweiligen strafbewerten Tat. Diese Voraussetzung besagt, dass das angewandte Mittel erstens zur Abwendung der Gefahr geeignet und zweitens unter mehreren etwa zur Verfügung stehenden Mitteln das mildeste, d.h. fremde Rechtsgüter am wenigsten beeinträchtigende sein muss

34 Urteil BGL, S. $7 \mathrm{ff}$.

35 Urteil BGL, S. 51 f., 54 .

36 Dass die Schäden - namentlich klimabedingte Beeinträchtigungen der Gesundheit bis hin zum Tode, die Zerstörung von Fahrnis- und Immobilieneigentum usw. - erst in der (näheren oder ferneren Zukunft) eintreten, steht der Unmittelbarkeit der Gefahr nicht entgegen, geht es doch nur darum, ob ein sofortiges Handeln erforderlich ist, um den zukünftig drohenden Schaden abzuwenden. (sog. Grundsatz der strikten/absoluten Subsidiarität). 37 Massgebend ist wiederum das ex ante-Urteil eines verständigen Dritten in der Lage des Täters. ${ }^{38}$ An die Geeignetheit des Mittels sind strenge Massstäbe anzulegen, damit nicht nutzlos in fremde Rechtsgüter eingegriffen wird. 39 Als ungeeignet sind solche Handlungen von vornherein auszuscheiden, durch welche die Rettungschancen nicht oder nur ganz unwesentlich erhöht werden. 40

16 Das Bezirksgericht Lausanne bejahte im hier besprochenen Fall das Vorliegen einer nicht anders abwendbaren Gefahr, nachdem es vier in Betracht kommende Handlungsalternativen als aussichtslos einstufte: 41

\section{Eine Protestaktion auf öffentlichem} Grund wäre nicht bewilligt worden und hätte, falls doch, nicht die ge-

37 Vgl. Urteil des Bundesgerichts 6B_495/2016 vom 16. Februar 2017 E. 2.2.2; Donatsch/Tag (Fn. 14), S. 242 f.; Dupuis et al. (Fn. 14), Art. 17 N 8; CR CP I-Monnier (Fn. 14), Art. 17 N 10; BSK StGB INiggli/Göhlich (Fn. 14), Art. 17 N 16; zum deutschen Recht S/S-K dStGB-Perron (Fn. 13), § 34 N 18 ff.; Claus Roxin, Strafrecht Allgemeiner Teil, Bd. I, 4. Aufl., München 2006, § 16 N 23.

38 Jakobs (Fn. 23), 13. Abschn. N 16; Mona/Leu (Fn. 25), 186 f.; Seelmann/Geth (Fn. 14), N 166; ähnlich Donatsch/Tag (Fn. 14), S. 243.

39 Frister (Fn. 13), 17. Kap. N 7; Kühl (Fn. 23), § 8 N 80; Roxin, Strafrecht Allgemeiner Teil (Fn. 37), $\S 16 \mathrm{~N} \mathrm{23}$; anders Nussbaumer (Fn. 16).

40 Volker Erb, in: Wolfgang Joecks/Klaus Miebach (Hrsg.), Strafgesetzbuch, Münchener Kommentar, Bd. 1, 3. Aufl., München 2017, § 34 N 91 (zit. MK dStGB-Autor); Kühl (Fn. 23), § 8 N 81; Mona/Leu (Fn. 25), S. 187; S/S-K dStGB-Perron (Fn. 13), § 34 N 19; Richter (Fn. 23), S. 128; Seelmann/Geth (Fn. 14), N 166; Frank Zieschang, in: Gabriele Cirener et al. (Hrsg.), Strafgesetzbuch, Leipziger Kommentar, Bd. III, 13. Aufl., Berlin 2019, § 34 N 91 (zit. LK dStGB-Autor); allerdings ist ein Mittel nicht schon deshalb ungeeignet, weil es den Schaden nicht mit Sicherheit oder mit hoher Wahrscheinlichkeit abwenden kann (Trechsel/Noll/Pieth [Fn. 14], S. 116 f.).

41 Urteil BGL, S. 52 ff., E. 4.2. 
wünschte und vorliegend auch tatsächlich erreichte Aufmerksamkeit erzielt.

2. Ebenso wenig hätte eine formelle Kontaktaufnahme mit der Bank, damit diese die Kritik zur Kenntnis nimmt und klimafreundliche Vorsätze fasst, Aussicht auf Erfolg gehabt, zumal die Angeklagten sowie die Organisation Greenpeace dieses Mittel bereits ausgeschöpft hatten.

3. Weiter wäre das Beschreiten des politischen Wegs nicht erfolgversprechend gewesen. Dies zeige sich namentlich daran, dass schon seit fünf Jahren verschiedene Parlamentarier den Bundesrat mit Interpellationen auf die unmittelbar drohende Klimakatastrophe hingewiesen haben, jedoch ohne von diesem dadurch mehr als «des déclarations d'intention inoffensives, voire lénifiantes, contredites par la réalité financière, scientifique ou politique» hervorzurufen.

4. Endlich wäre die Bestreitung des Rechtswegs nicht verheissungsvoll gewesen, weil die einschlägigen Rechtsgrundlagen (Art. 73 f. BV, Art. 2 und 8 EMRK, das Pariser Klimaabkommen) $\mathrm{zu}$ wenig respektiert würden und rechtlich nicht durchzusetzen seien.

Ungeachtet dessen, ob man die Einschätzung des Bezirksgerichts Lausannes bezüglich der alternativen Handlungsmöglichkeiten teilt, ist dessen Argumentation in einem entscheidenden Punkt zu bemängeln: Es wird nicht klar differenziert zwischen dem Nahziel der Klimaaktivisten, die öffentliche Aufmerksamkeit auf die Problematik des Klimawandels und
Investitionen der Crédit Suisse in fossile Brennstoffe zu lenken, und dem Endziel, die Erderwärmung auf höchstens $1.5^{\circ} \mathrm{C}$ über dem vorindustriellen Niveau zu begrenzen bzw. einen dramatischen Klimawandel und die damit verbundenen Beeinträchtigungen von Individualrechtsgütern (vgl. oben II.B.) zu verhindern. ${ }^{42}$ Rechtlich gesehen kann im Lichte des Art. 17 StGB nur Letzteres relevant sein; $\mathrm{zu}$ fragen ist somit, ob die vorliegende Protestaktion geeignet und das mildeste Mittel war, die vom Klimawandel ausgehende Gefahr für Rechtsgüter des Einzelnen (zumindest teilweise) abzuwehren.

18 Das kann im hier besprochenen Fall aber nicht als nachgewiesen gelten. Selbst wenn eine verständige Drittperson in der Lage der Aktivisten davon ausgehen konnte, dass die Aktion geeignet war, ein grosses Medienecho auszulösen und vielleicht sogar eine Stellungnahme von Roger Federer zu veranlassen, so war auch klar, dass damit das Klimaproblem bei Weitem nicht gelöst wäre. Es genügt nicht zu sagen, dass man mit einer Sensibilisierungsaktion vielleicht einen Bruchteil eines Promilles näher an die Lösung der globalen Klimafrage herankomme - eine solche Argumentation führt ins Grenzenlose. Das Notstandserfordernis der Eignung ist im vorliegenden Fall somit nicht erfüllt. 43 Dies dürfte auch bei anderen Klima-Protestaktionen regelmässig der Fall sein: Ein Tropfen auf den heissen Stein genügt den Anfor-

$42 \overline{\text { Vgl. die Stellungnahmen einiger Beschuldigten }}$ anlässlich der Pressekonferenz vom 28. November 2019; sowie Urteil BGL, S. $25 \mathrm{ff.}$

43 So auch Schild Trappe/Schöbi (Fn. 14), N 17 und 20. 
derungen der Eignung des Tatverhaltens zur Gefahrenabwehr nicht. 44

\section{Zwischenfazit}

19 Aufgrund des vorstehend Dargelegten ist davon auszugehen, dass bei Straftaten, die im Rahmen von Klima-Protestaktionen begangen werden, die Anwendung des rechtfertigenden Notstands im Sinne von Art. 17 StGB regelmässig am Erfordernis der Eignung des Tatverhaltens zur Abwehr der Gefahr scheitert. Ferner wird in diesen Fällen eine Entschuldigung oder Strafmilderung wegen entschuldbaren Notstands (Art. 18 StGB) am gleichen Erfordernis scheitern, da auch Art. 18 StGB eine «nicht anders abwendbare Gefahr» bzw. eine zur Gefahrenabwehr geeignete und erforderliche Notstandshandlung voraussetzt. 45

\section{Aussergesetzlicher Notstand}

2o Zur Wahrung von Rechtsgütern der Allgemeinheit, welche durch seine Beschränkung auf Individualrechtsgüter nicht von Art. 17 StGB erfasst werden (vgl. oben II.A.), wird ein «aussergesetzlicher Notstand» (auch «übergesetzlicher Notstand» genannt46) diskutiert.47 Der

44 Vgl. die Nachweise in Fn. 39 f. sowie Jakobs (Fn. 23), 13. Abschn. N 17: «Ein der Art nach brauchbares, der Menge nach jedoch zu schwaches Mittel ist ungeeignet; die Gefahr ist so nicht abwendbar; Beispiel: Ein Handfeuerlöscher ist zur Bekämpfung eines Großbrands ungeeignet.»

45 Vgl. Art. 18 Abs. 1 StGB; Anna Coninx, Der entschuldigende Notstand zwischen Unrecht und Schuld, ZStrR 2013, S. 113 ff., 118 f., 122.

46 Krit. zu dieser Terminologie BSK StGB INiggli/Göhlich (Fn. 14), Art. 17 N 6; Schultz (Fn. 14), S. 149 f., 175; vgl. auch Graven/Sträuli (Fn. 14), N 102.

47 Vgl. BGE 94 IV 68 E. 2; Germann (Fn. 14), vor Art. 32 N 8; Graven/Sträuli (Fn. 14), N 102; Iwangoff (Fn. 14), S. 144 f.; Horst Schröder, Die Not als Rechtfertigungs- und Entschuldigungsgrund im deutschen und schweizerischen Straf- mögliche Anwendungsbereich dieses Rechtfertigungsgrundes wird von seinen Befürwortern allerdings stark begrenzt, nämlich auf den Schutz der «Lebensinteressen des Staats», den höchsten Gütern eines Landes, wie seinem Bestand, seiner Unabhängigkeit, territorialen Integrität usw. ${ }^{48}$ Des Weiteren wird die Einhaltung der anderen Voraussetzungen des rechtfertigenden Notstandes nach Art. 17 StGB verlangt.49 Schliesslich wird vielfach betont, dass Private von vornherein nicht befugt sind, «für den Staat» zu handeln, 50 sie sich also auf den aussergesetzlichen Notstand nicht berufen können. ${ }^{51}$ Teilweise wird diese letzte Aussage allerdings dahingehend relativiert, dass privates Handeln zur Wahrung der höchsten Güter des Staates jedenfalls absolut subsidiär zum Eingreifen der zuständigen Behörde erfolgen müsse, damit eine Rechtfertigung in Betracht kommt.52

recht, ZStrR 1960, S. 1 ff., 12 ff.; Seelmann/Geth (Fn. 14), N 182; Stratenwerth (Fn. 10), § 10 N 57 f.; ablehnend BSK StGB I-Niggli/Göhlich (Fn. 14), Art. 17 N 6.

48 Stratenwerth (Fn. 10), § 10 N 58, m.Verw. auf Reichsgericht in Strafsachen 63, 215, S. 220; vgl. auch Iwangoff (Fn. 14), S. 144; Waiblinger (Fn. 16), Ziff. 7.

49 Seelmann/Geth (Fn. 14), N 182, m.H. auf BGE 94 IV 68 E. 2; Stratenwerth (Fn. 10), § 10 $\mathrm{N} 58$.

50 Eine solche Befugnis kann sich hingegen aus dem Gesetz ergeben, wie etwa beim strafprozessualen Festnahmerecht Privater gemäss Art. 218 StPO (so auch Arzt [Fn. 13], S. 29).

51 Vgl. Arzt (Fn. 13), S. 29; Iwangoff (Fn. 14), S. 144; BSK StGB I3-Kurt Seelmann, in: Marcel Alexander Niggli/Hans Wiprächtiger (Hrsg.), Strafrecht I, Basler Kommentar, 3. Aufl., Basel 2013, Art. 17 N 15 (zit. BSK StGB I3-Autor); dens./Geth (Fn. 14), N 182; Waiblinger (Fn. 16), Ziff. 7; vgl. auch BSK StGB I-Niggli/Göhlich (Fn. 14), vor Art. 14 N 68, 71.

52 Vgl. BSK StGB I3-Seelmann (Fn. 51), Art. 17 N 15; ders./Geth (Fn. 14), N 182; Stratenwerth (Fn. 10), $\S 10 \mathrm{~N}$ 58; vgl. auch BGE 129 IV 6 E. 3.1; Waiblinger (Fn. 16), Ziff. 7; vgl. zum deutschen Recht HansHeinrich Jescheck/Thomas Weigend, Lehrbuch des Strafrechts, Allgemeiner Teil, 5. Aufl., Berlin 
21 Ein solcher aussergesetzlicher Notstand vermag die Klimaaktivisten im vorliegend besprochenen Fall nicht zu entlasten. Selbst wenn man Klima- und Umweltschutz als Rechtsgüter der Allgemeinheit betrachten würde, 53 welche zu den «vitalen Interessen» des Staates gehören, oder man im Klimawandel eine unmittelbare Gefahr für den Bestand des Gemeinwesens erblicken würde, und man sich weiter wie das Bezirksgericht Lausanne auf den Standpunkt stellen würde, dass der Staat zum Schutz dieser Güter weitgehend untätig bleibe und ein Einschreiten durch Private folglich nicht a priori ungerechtfertigt sei, 54 so sind die weiteren Voraussetzungen des rechtfertigenden Notstands, wie namentlich die Eignung der Notstandstat zur Abwendung der Gefahr für das Erhaltungsgut, nicht erfüllt (vgl. oben II.C.). Eine Rechtfertigung gestützt auf den aussergesetzlichen Notstand dürfte deshalb auch bei anderen im Zusammenhang mit Klimaprotesten begangenen Straftaten nicht einzuräumen sein.

\section{Wahrnehmung berechtigter Interessen}

Ein weiterer aussergesetzlicher Rechtfertigungsgrund, die «Wahrnehmung berechtigter Interessen» (auch "Wahrung berechtigter Interessen» genannt), findet in der bundesgerichtlichen Rechtsprechung durchwegs Anerkennung.55 Das

1996, S. 340 f.; Johannes Wessels/Werner Beulke/ Helmut Satzger, Strafrecht Allgemeiner Teil, 48. Aufl., Heidelberg 2018, N 459.

53 Vgl. zur Kontroverse Fn. 18.

54 Vgl. Urteil BGL, S. 54.

55 Vgl. BGE 134 IV 216; BGE 129 IV 6; BGE 127 IV 166; BGE 127 IV 122; BGE 126 IV 236; BGE 120 IV 208; BGE 117 IV 170; BGE 115 IV 75; BGE 113 IV 4; BGE 94 IV 68; Urteile des Bundesgerichts 6B_1267/2015 vom 25. Mai 2016; 6B_186/2012
Bundesgericht räumt jedoch ein, dass bei einem Rechtfertigungsgrund unter diesem Titel die Gefahr besteht, «dass unter pauschaler Berufung auf schutzbedürftige private oder öffentliche Interessen der strafrechtliche Rechtsgüterschutz ausgehöhlt und unterlaufen werden könnte». 56 Voraussetzung für den Rechtfertigungsgrund der Wahrnehmung berechtigter Interessen ist daher grundsätzlich, dass zuvor der Rechtsweg mit legalen Mitteln beschritten und ausgeschöpft worden ist.57 Im Übrigen wird - in Angleichung an die Erfordernisse des rechtfertigenden Notstands - verlangt, dass «die Tat ein notwendiges und angemessenes Mittel ist, um ein berechtigtes Ziel zu erreichen, sie insoweit den einzig möglichen Weg darstellt und offenkundig weniger schwer wiegt als die Interessen, welche der Täter zu wahren sucht». $5^{8}$ Bei der Prüfung dieser Voraussetzungen sind strenge Massstäbe anzulegen:59 «Essa deve essere interpretata restrittivamente con esigenze particolarmente severe per quanto concerne la valutazione della sussidiarietà e

vom 11. Januar 2013; 6B_305/2011 vom 12. Dezember 2011; 6B_225/2008 vom 7. Oktober 2008; 6S.256/2002 vom 26. Oktober 2002; zur Entstehungsgeschichte vgl. BGE 85 IV 182 (m.w.H.).

56 BGE 129 IV 6 E. 3.3; in diesem Sinne auch Arzt (Fn. 13), S. 31; Donatsch/Tag (Fn. 14), S. 264; Franz Riklin, Zum Rechtfertigungsgrund der Wahrung (Wahrnehmung) berechtigter Interessen, in: Andreas Donatsch/Marc Forster/Christian Schwarzenegger (Hrsg.), Strafrecht, Strafprozessrecht und Menschenwürde, Festschrift für Stefan Trechsel, Zürich 2002, S. 537 ff., 546.

57 BGE 129 IV 6 E. 3.1 und 3.3; BGE 115 IV 75 E. 4b; BGE 94 IV 68 E. 2.

58 BGE 134 IV 216 E. 6.1 m.w.N.

59 Vgl. BGE 134 IV 216 E. 6; BGE 129 IV 6 E. 3.5; BGE 127 IV 166 E. 2b und 2f; BGE 120 IV 208 E. 3b; BGE 115 IV 75 E. 4b; BGE 94 IV 68 E. 2; BGE 70 IV 20 E. 2; Urteil des Bundesgerichts 6B_305/2011 vom 12. Dezember 2011, E. 4, Bem. Matthias Schwaibold, FP 2012, S. 66 ff., 70; vgl. auch Graven/Sträuli (Fn. 14), N 112. 
della proporzionalità.» ${ }^{60}$ Endlich ist nach der Rechtsprechung des Bundesgerichts eine Berufung auf die Wahrnehmung berechtigter Interessen nicht möglich, wenn das geltende Recht einen Konflikt schon abschliessend entschieden hat. ${ }^{61}$

Unklar bleibt nach der bundesgerichtlichen Definition, was ein berechtigtes Ziel darstellt. Im Schrifttum hat man versucht, die Fälle, in denen das Bundesgericht die Wahrnehmung berechtigter Interessen immerhin erwog, auf einen gemeinsamen Nenner zu bringen. Demnach geht es bei der Wahrnehmung berechtigter Interessen um die Ausübung verfassungsmässig garantierter Freiheitsrechte sowie die Herstellung sozial erwünschter und gebilligter Zustände auf Kosten der Beeinträchtigung anderer Interessen. ${ }^{62}$ Mit anderen Worten erscheint ein Ziel jedenfalls dann als berechtigt, wenn es sozial erwünscht oder grundrechtlich geschützt ist. ${ }^{63} \mathrm{Um}$ die Regelung des Art. 17 StGB nicht zu unterlaufen bzw. eine Abgrenzung zum aussergesetzlichen Notstand zu verunmöglichen,

6o BGE 127 IV 166 E. 2b.

61 BGE 120 IV 208 E. 3a; unveröffentl. Urteil des Bundesgerichts vom 22. August 1990, zit. in: BGE 117 IV 170 E. 3b.

62 Vgl. Donatsch/Tag (Fn. 14), S. 264 ff.; Dupuis et al. (Fn. 14), Art. 14 N 36; Germann (Fn. 14), vor Art. 32 N 9; CR CP I-Monnier (Fn. 14), Art. 14 N 55; BSK StGB I-Niggli/Göhlich (Fn. 14), vor Art. 14 N 66; Peter Noll, Die Rechtfertigungsgründe im Gesetz und in der Rechtsprechung, ZStrR 1964, S. 160 ff., 188; dens., Tatbestand und Rechtswidrigkeit: Die Wertabwägung als Prinzip der Rechtfertigung, ZStW 1965, S. 1 ff., 32; Riklin (Fn. 14), § 14 N 70; Seelmann/Geth (Fn. 14), N 183; Stratenwerth (Fn. 10), § 10 N 59 ff.; PK StGB-Trechsel/Geth (Fn. 14), Art. 14 N 13; Trechsel/Noll/Pieth (Fn. 14), S. 134.

63 In diesem Sinne auch Albin Eser, Wahrnehmung berechtigter Interessen als allgemeiner Rechtfertigungsgrund, Bad Homburg v.d.H./Berlin/Zürich 1969, S. 44 f.; enger (beides kumulativ verlangt) Noll, ZStrR (Fn. 62), S. 188. ist nach Stratenwerth ${ }^{64}$ weiter die Anwendung des Rechtfertigungsgrundes der Wahrnehmung berechtigter Interessen auf konkret bedrohte, schon bestehende Rechtsgüter des Einzelnen oder der Allgemeinheit ausgeschlossen. Ansonsten könne «wohl jedes schutzwürdige private oder öffentliche Interesse in Betracht kommen».

24 Im hier besprochenen Fall ist zunächst fraglich, worin das wahrgenommene berechtigte Interesse liegen soll. Etwa in der Vermeidung eines dramatischen Klimawandels? Hier scheitert es jedoch am Kriterium der Eignung bzw. Notwendigkeit der Tat zur Wahrnehmung des berechtigten Ziels (vgl. oben II.C.).65 Zum selben Ergebnis gelangt man, wenn man das berechtigte Interesse in der Informierung der Öffentlichkeit über die Problematik des Klimawandels und Investitionen der Bank in fossile Brennstoffe erblicken will: Erlaubte Demonstrationen, Zeitungsanzeigen und Medienkampagnen können diesen Anliegen ebenso Gehör verschaffen; hier gilt es, die restriktive Rechtsprechung des Bundesgerichts $\mathrm{zu}$ beachten, welche besonders strenge Anforderungen an die Voraussetzungen der Subsidiarität und Verhältnismässigkeit stellt. Schliesslich fällt die Verhinderung klimaschädlicher Investitionen und Finanzierungen durch die Bank bzw. andere Finanzmarktakteure nicht unter die Wahrnehmung berechtigter Interessen. Denn diesbezüglich kann

64 Stratenwerth (Fn. 10), § 10 N 57 ff., 6o f.; vgl. auch Iwangoff (Fn. 14), S. 143; HK StGB-Wohlers (Fn. 14), Art. $17 \mathrm{~N} 11$.

65 Zudem müssten, damit nach Stratenwerth überhaupt ein berechtigtes Ziel vorliegt, der Klimaund Umweltschutz nicht bereits (konkret bedrohte, schon existierende) Rechtsgüter darstellen, da ansonsten einzig der (aussergesetzliche) Notstand einschlägig wäre. 
nicht gesagt werden, die politischen und rechtlichen Möglichkeiten seien ausgeschöpft worden. Den Klimaaktivisten steht es zusätzlich zu den genannten Manifestationsmöglichkeiten beispielsweise offen, eine Volksinitiative zu lancieren, um die Wirtschaftsfreiheit am Schweizer Finanzmarkt bezüglich solchen Investitionen und Finanzierungen 66 einzuschränken. Selbst wenn man dem entgegenhalten wollte, dass ein solches Vorgehen einige Zeit in Anspruch nehmen würde und der Zeitfaktor vorliegend eine wesentliche Rolle spielt, ${ }^{67}$ wird eine Rechtfertigung mangels Geeignetheit der Tat zur Erreichung des besagten Ziels scheitern müssen.

Es bleibt das Interesse der Aktivisten an der Ausübung der Meinungs- und Versammlungsfreiheit, deren Schutzbereiche im besprochenen Fall eröffnet sind. Zwar könnte dies im Hinblick auf Art. 22 BV mit der Begründung bestritten werden, dass gegen Straftatbestände verstossende Versammlungen nicht friedlich seien und deshalb aus dem Schutzbereich der Be-

$66 \overline{\text { Gegenwärtig setzt der Bundesrat bei der Zieler- }}$ reichung, die Finanzflüsse klimaverträglich auszugestalten, in einem ersten Schritt auf freiwillige Massnahmen der Finanzmarktakteure. Sollten freiwillige Massnahmen und gesteigerte Transparenz jedoch ungenügende Wirkung zeigen, wird der Bundesrat weitere Massnahmen zur Zielerreichung in Betracht ziehen (vgl. Botschaft vom 1. Dezember 2017 zur Totalrevision des $\mathrm{CO}_{2}-$ Gesetzes nach 2020, BBl 2018247 ff., S. 249, 260, 269 f.; Stellungnahme des Bundesrates vom 29. Mai 2019 zur Interpellation von Beat Vonlanthen 19.3230; vgl. ferner Mirjam Eggen, Klimaverträgliche Finanzflüsse, Jusletter 16. März 2020, N 6; Rolf H. Weber/Andreas Hösli, Der Klimawandel und die Finanzmärkte, GesKR 2019, S. 574 ff., 580 f.). Im Zusammenhang mit Regulierungsbemühungen, vgl. Geschäft des Bundesrates 17.071, Totalrevision des CO2Gesetzes nach 2020.

67 Vgl. BGE 117 IV 170 E. 3c; Donatsch/Tag (Fn. 14), S. 265. stimmung von vornherein herausfielen. Doch ist Unfriedlichkeit nicht mit Rechtswidrigkeit gleichzusetzen, sondern nur dann anzunehmen, wenn die Veranstalter oder die Teilnehmer Gewaltakte gegen Leib, Leben, Freiheit oder Eigentum Dritter beabsichtigen, ${ }^{68}$ was vorliegend nicht der Fall war.

26 Allerdings unterliegen die Grundrechte der Meinungs- wie der Versammlungsfreiheit der Beschränkung durch Gesetze (Art. $36 \mathrm{BV}$ ), also auch durch Strafgesetze. Will man trotzdem zu einer Wahrnehmung berechtigter Interessen, gestützt auf die Ausübung verfassungsmässig garantierter Freiheitsrechte kommen, so muss man auf das Prinzip zurückgreifen, wonach die beschränkenden Gesetze im Lichte der Grundrechte auszulegen sind, sodass eine Abwägung zwischen Grundrecht und Strafgesetz stattzufinden hat. 69

27 Das Ergebnis der Abwägung wird im vorliegenden Fall freilich gegen eine Rechtfertigung ausfallen müssen. Denn die Freiheit des Einzelnen endet dort, wo das

68 Vgl. BGE 132 I 256 E. 3; Christoph Errass, in: Bernhard Ehrenzeller et al. (Hrsg.), Die schweizerische Bundesverfassung, St. Galler Kommentar, 3. Aufl., Zürich et al. 2014, Art. 22 N 19 ff. (zit. SGK BV-Autor); Maya Hertig, in: Bernhard Waldmann/Eva Maria Belser/Astrid Epiney (Hrsg.), Bundesverfassung, Basler Kommentar, Basel 2015, Art. 22 N 8 m.w.H. (zit. BSK BVAutor); vgl. auch Urteil des EGMR 29225/95 vom 2. Oktober 2001 (Stankov and the United Macedonian Organisation Ilinden v. Bulgaria); Urteil des deutschen Bundesverfassungsgerichts 73, 206, S. $248 \mathrm{f}$.

69 Vgl. BGE 134 IV 216 E. 5.2.2 und 5.3; BGE 127 IV 166 E. 2g; BGE 118 IV 153 E. 4c; BGE 116 IV 31 E. 5a/bb; BGE 106 Ia 33 E. 2 und 3; Patricia Egli, Drittwirkung von Grundrechten, Diss. Zürich 2002, S. 193; BSK BV-Epiney (Fn. 68), Art. 190 N 22; BSK BV-Hertig (Fn. 68), Art. 22 N 34; Regina Kiener/Walter Kälin, Grundrechte, 2. Aufl., Bern 2013, S. 44; in Deutschland (vgl. Urteil des deutschen Bundesverfassungsgerichts 7,198) als «Wechselwirkungstheorie» bezeichnet. 
Recht des anderen beginnt. ${ }^{70}$ Dem Interesse der Aktivisten an der Ausübung der Meinungs- und Versammlungsfreiheit stehen nebst öffentlichen Interessen das Interesse der Bank am ungestörten Geschäftsbetrieb und die (strafrechtlich geschützte) Freiheit, selbst zu bestimmen, wer sich in den Filialräumlichkeiten aufhalten darf (sog. Hausrecht), gegenüber. Im Übrigen muss der Schutz der Rechte der Bank auch vor dem Hintergrund der staatlichen Schutzpflichten gesehen werden. Schliesslich ist zu berücksichtigen, dass bewusste Normverletzungen als Mittel einer Minderheit, auf den öffentlichen Willensbildungsprozess einzuwirken, mit den Grundprinzipien des demokratischen Rechtsstaates schlechterdings unvereinbar sind. ${ }^{71}$

Andernfalls wäre ja nicht einmal das Wegtragen der Aktivisten durch die Polizei zulässig, sondern würde als rechtswidriger Angriff deren Notwehrrecht begründen; denn auch der Widerstand gegen polizeiliche Anordnungen müsste dadurch neutralisiert werden. ${ }^{72}$ Darin läge eine Selbstaufgabe der Demokratie, ein Verzicht auf die Durchsetzung der

70 Vgl. Immanuel Kant, Die Metaphysik der Sitten, Königsberg 1797, passim.; § 83 Allgemeines Landrecht für die Preußischen Staaten vom 5. Februar 1794, Einleitung; Art. 4 Déclaration des droits de l'homme et du citoyen vom 26. August 1789.

${ }_{71}$ S/S-K dStGB-Perron (Fn. 13), § 34 N 41a; in diesem Sinne auch BGE 129 IV 6 E. 3.1; Ulrich Karpen, «Ziviler Ungehorsam» im demokratischen Rechtsstaat, JZ 1984, S. 249 ff., 259, 262; ähnlich Claus Roxin, Strafrechtliche Bemerkungen zum zivilen Ungehorsam, in: Peter-Alexis Albrecht et al. (Hrsg.), Festschrift für Horst SchülerSpringorum zum 65. Geburtstag, Köln et al. 1993, S. $441 \mathrm{ff}$., $446,448$.

72 So auch Schild Trappe/Schöbi (Fn. 14), N 10 f.
Mehrheitsregel, der rechtlich unmöglich ist.73

29 Somit vermag auch der Rechtfertigungsgrund der Wahrnehmung berechtigter Interessen - ungeachtet seiner fraglichen Legitimation und Tragweite überhaupt 74 - in diesem und ähnlichen Fällen keine Rechtfertigung herbeizuführen.

\section{Zur Möglichkeit des Absehens von der Strafe}

30 Mit der Erkenntnis, dass in Fällen der vorliegenden Art weder strafgesetzliche Notstandsinstitute noch aussergesetzliche, notstandsähnliche Rechtfertigungsgründe die Unrechtmässigkeit der verübten Straftaten auszuschliessen vermögen, ist die strafrechtliche Problematik aber noch nicht gelöst. Denn es ist eine Frage, ob man im Rahmen von Klimaprotesten begangene Straftaten für rechtmässig erklärt, und eine andere, ob man sie in jedem Fall bestrafen muss.

31 Der schweizerische Gesetzgeber hat, um Fälle relativ unbedeutender Verhaltensweisen, welche die Schwere und Härte einer Strafe nicht verdienen, den Strafbefreiungsgrund des fehlenden Strafbedürfnisses (Art. 52 StGB) geschaffen.75 Dessen Anwendung setzt zunächst geringfügige Schuld und Tatfolgen voraus. Ausserdem ist erforderlich, dass spezialoder generalpräventive Strafzwecke eine Bestrafung nicht notwendig machen. ${ }^{76}$

$73 \overline{\text { Vgl. Roxin, Strafrechtliche Bemerkungen (Fn. 71), }}$ S. 448.

74 Vgl. BSK StGB I-Niggli/Göhlich (Fn. 14), vor Art. $14 \mathrm{~N} 67 \mathrm{ff}$.

75 Botschaft (Fn. 12), BBl 19991979 ff., S. 2063.

76 Vgl. BSK StGB I-Riklin (Fn. 14), Art. 52 N 26; Günter Stratenwerth/Felix Bommer, Schweizerisches Strafrecht, Allgemeiner Teil II: Strafen und 

das Vorliegen geringfügiger Schuld, ist in Fällen wie im besprochenen zweifellos erfüllt. Denn die Schuld ist in zweifacher Weise gegenüber dem Regelfall der Straftat drastisch reduziert. Erstens ist schon objektiv das Unrecht und damit die seiner vorsätzlichen Verwirklichung entsprechende Schuld gering. Zweitens ist auch subjektiv die schuldrelevante Motivation der Täter gemeinwohlorientiert, so dass der gleichwohl zu missbilligende Gesetzesverstoss nur eine sehr geringe «Vorwerfbarkeit» übrig lässt.

Des Weiteren kann auch die Geringfügigkeit der Tatfolgen als gegeben betrachtet werden, nämlich unter dem Aspekt der «Wertungskongruenz». Denn die vorliegenden Auswirkungen der Tat divergieren beträchtlich von denjenigen, die der Gesetzgeber als schwere Tatfolgen vor Augen hatte (Tod und schwere Körperverletzung).77

34 Schliesslich ist in Fällen der vorliegenden Art eine Bestrafung weder spezial- noch generalpräventiv erforderlich. Spezialpräventiv nicht, weil die Klimaaktivisten um das Gemeinwohl besorgte Bürger und keine «Verbrecher» sind,78 und weil sozialpolitisch die Missbilligung des Regelverstosses durch Beharren auf seine Rechtswidrigkeit (mit der Folge einer etwaigen Schadenersatzpflicht) genügt. Eine weitere Missbilligung durch die Verhängung einer Strafe (mit der Konsequenz eines Strafregistereintrags,

Massnahmen, 3. Aufl., Bern 2020, § 6 N 5; PK StGB-Trechsel/Keller (Fn. 14), Art. 52 N 2 b.

77 Botschaft (Fn. 12), BBl 19991979 ff., S. 2064.

78 In Anlehnung an Horst Schüler, Strafrechtliche Aspekte zivilen Ungehorsams, in: Peter Glotz (Hrsg.), Ziviler Ungehorsam im Rechtsstaat, Frankfurt a.M. 1983, S. 76 ff., 82.
Art. 366 Abs. 2 lit. a StGB) ist spezialpräventiv kontraindiziert, weil sie geeignet ist, die Täter in eine Radikalisierung zu treiben, die sie dazu führen könnte, von symbolischen Regelverstössen zu wirklich kriminellen Verhaltensweisen überzugehen. Eine Bestrafung ist ferner unter dem Gesichtspunkt der Generalprävention nicht erforderlich, weil es in ihrer heute herrschenden Form nicht um Abschreckung, sondern vor allem um soziale Konfliktbereinigung geht,79 die in geringfügigen Fällen wie vorliegend besser durch einen Strafverzicht als durch eine Bestrafung zu erreichen ist.

35 Somit ist Fällen wie dem vorliegenden gestützt auf Art. 52 StGB von einer Bestrafung abzusehen. Es erscheint allerdings notwendig, die vorstehenden Ausführungen noch zu präzisieren und einen Verzicht auf Strafe bei solchen KlimaProtestaktionen nur zuzulassen, wenn:

1. die Protestierenden aus Sorge um das Gemeinwohl handeln;

2. sie sich eindeutig zur halbdirekten Demokratie bekennen;

3. der Gesetzesverstoss einen ersichtlichen Zusammenhang mit dem Adressaten des Protestes aufweist;

4. die Gesetzesverletzung alle Gewalttätigkeit und den Widerstand gegen Ordnungskräfte meidet;

5. die aus dem Protest erwachsende Behinderung und Belästigung geringfügig und zeitlich begrenzt bleibt. 80

79 Roxin, Strafrecht Allgemeiner Teil (Fn. 37), § 3 N 27; Stratenwerth (Fn. 10), § 2 N 23.

80 Ähnlich Roxin, Strafrechtliche Bemerkungen (Fn. 71), S. 456; ders. (Fn. 37), § 22 N 133. 
36 Wird unter diesen Voraussetzungen eine Strafbefreiung (Art. 52 StGB) zugebilligt, so kann damit den Umständen des Einzelfalles genügend Rechnung getragen werden, ohne dass daraus dieselben dogmatischen Ungereimtheiten resultieren wie bei der Anwendung des rechtfertigenden Notstands oder notstandsähnlicher Rechtfertigungsgründe. 